\title{
Finite groups whose maximal subgroups of order divisible by all the primes are supersolvable
}

\author{
by \\ Alexander Moretó \\ Departament de Matemàtiques \\ Universitat de València \\ 46100 Burjassot. València SPAIN \\ Alexander.Moreto@uv.es
}

\begin{abstract}
We study finite groups $G$ with the property that for any subgroup $M$ maximal in $G$ whose order is divisible by all the prime divisors of $|G|, M$ is supersolvable. We show that any nonabelian simple group can occur as a composition factor of such a group and that, if $G$ is solvable, then the nilpotency length and the rank are arbitrarily large. On the other hand, for every prime $p$, the $p$-length of such a group is at most 1 . This answers questions proposed by V. Monakhov in The Kourovka Notebook.
\end{abstract}

AMS Subject Classification. 20D10, 20F16

Keywords and phrases. supersolvable subgroup, maximal subgroup, simple group, solvable group, $p$-length, Fitting height

Research supported by Ministerio de Ciencia e Innovación PID-2019-103854GB100, FEDER funds and Generalitat Valenciana AICO/2020/298. 


\section{Introduction}

Problem 19.55 in The Kourovka Notebook [3], proposed by V. Monakhov, asks the following.

Problem. Suppose that in a finite group $G$ every maximal subgroup $M$ is supersolvable whenever $\pi(M)=\pi(G)$, where $\pi(G)$ is the set of all prime divisors of the order of $G$.

(i) What are the nonabelian composition factors of $G$ ?

(ii) Determine exact upper bounds for the nilpotency length, the p-length and the rank of $G$ if $G$ is solvable.

The goal of this note is to answer these questions. First, we show that any nonabelian simple group can occur as a composition factor of some group with this property. It is perhaps remarkable that we do not need the classification of finite simple groups to prove this.

Theorem A. Every nonabelian simple group can occur as a composition factor of a finite group with the property that every maximal subgroup $M$ of $G$ with $\pi(M)=\pi(G)$ is supersolvable.

Regarding the second part of Monakhov's question, we show that there is not any bound for nilpotency length and the rank, but the $p$-length is at most 1 for every prime $p$.

Theorem B. There exist solvable groups of arbitrarily large nilpotency length and arbitrarily large rank with the property that every maximal subgroup $M$ of $G$ with $\pi(M)=\pi(G)$ is supersolvable.

Theorem C. Let $G$ be a solvable group such that for every maximal subgroup $M$ of $G$ such that $\pi(M)=\pi(G), M$ is supersolvable. Then the $p$ length of $G$ is 1 for every prime divisor $p$ of $|G|$.

Note that groups of $p$-length at most 1 for every prime $p$ are known to have a number of properties (see, for instance, VI.6 of [1]).

\section{$2 \quad$ Arbitrary groups}

In this section we prove Theorem A. We start by recalling two well-known lemmas.

Lemma 2.1. Let $G$ be a finite group and $N \unlhd G$. If $P$ is a Sylow $p$-subgroup of $G$, then $N_{G / N}(P N / N)=N_{G}(P) N / N$. 
Lemma 2.2. Let $A_{p}$ be the alternating group on $p$ letters, where $p$ is a prime. Let $P \in \operatorname{Syl}_{p}\left(A_{p}\right)$. Then $\left|N_{A_{p}}(P)\right|=\frac{p-1}{2} p$.

Now, we prove Theorem A.

Proof of Theorem A. Let $q$ be bigger than the largest prime divisor of $|S|$ and let $p$ be a prime such that $p>2 q$. Note that this implies that $\frac{p-1}{2} \geq q$. Let $G=S$ 乙 $H$, where $H=A_{p}$ permutes transitively $p$ copies of $S$. Let $P \in \operatorname{Syl}_{p}(H)$ so that $P$ is also a Sylow $p$-subgroup of $G$. Let $N=S \times \cdots \times S$ be the base group. By Lemmas 2.1 and 2.2.

$$
\left|N_{G}(P) N / N\right|=\left|N_{G / N}(P N / N)\right|=\left|N_{A_{p}}(P)\right|=\frac{p-1}{2} p .
$$

This implies that if $r$ is a prime such that $q \leq \frac{p-1}{2}<r<p$, then $r$ does not divide $\left|N_{G}(P)\right|$. Notice that by Bertrand's Postulate, such a prime exists.

Now, we will show that $G$ does not have any maximal supersolvable subgroup of order divisible by all the primes in $\pi(G)$. By way of contradiction, let $M$ be such a maximal supersolvable subgroup. Since $p$ divides $|M|$, we may assume that $P \leq M$. Since $M$ is supersolvable, $p$ is the largest prime divisor of $|M|$, and $P \in \operatorname{Syl}_{p}(M), P \unlhd M$. But then $M \leq N_{G}(P)$, so all the prime divisors of $|G|$ divide $\left|N_{G}(P)\right|$. This is a contradiction.

\section{Solvable groups}

We start with the proof of Theorem B. The key to our construction is the following well-known lemma.

Lemma 3.1. Let $G$ be a finite (complex) linear group of degree $n$. Let $p$ be a prime such that $e=\exp (G)$ divides $p-1$. Then $G$ acts faithfully and irreducibly on an elementary abelian group $V$ of order $p^{n}$.

Proof. Since $\mathbb{F}_{p}$ contains a primitive $\exp (G)$ th root of unity, Corollary 9.15 of [2] implies that $\mathbb{F}_{p}$ is a splitting field for $G$. By hypothesis, $G$ has a faithful (complex) irreducible character $\chi$ of degree $n$ and since $p$ does not divide $|G|$, Theorem 15.13 of [2] implies that $\chi$ is also an irreducible $p$-Brauer character. Since $\mathbb{F}_{p}$ is a splitting field for $G$, the natural module for this character is an elementary abelian group of order $p^{n}$. The result follows.

Proof of Theorem $B$. Now, let $G_{1}=V_{1}$ be a cyclic group of order $p_{1}$ for some prime $p_{1}$. By Lemma 3.1, $G_{1}$ acts faithfully and irreducibly on an elementary abelian $p_{2}$-group $V_{2}$ (of order $p_{2}$, in this case). Put $G_{2}=G_{1} \ltimes V_{2}$. As before, 
$G_{2}$ acts faithfully and irreducibly on some elementary abelian $p_{3}$-group $\mathrm{H}_{3}$ and we put $G_{3}=G_{2} \ltimes V_{3}$. Inductively, we define $G_{n+1}=G_{n} \ltimes V_{n+1}=$ $V_{1} \ldots V_{n+1}$ for $n \geq 1$. Notice that since $V_{n}$ is the unique minimal normal subgroup of $G_{n}$ for every $n \geq 1, G_{n}$ has faithful irreducible characters and these groups do exist by Lemma 3.1 .

Note that the nilpotency length of $G_{n}$ is $n$ for every $n$. Also, the rank of $V_{n}$ goes to infinity when $n$ goes to infinity (for instance, because the nilpotency length of a linear group over a finite field is bounded in terms of the dimension. See Theorem 3.9(b) of [4]).

It remains to see that the groups $G_{n}$ satisfy the hypothesis of the question. Clearly, we may assume $n>2$. Note that $\left|G_{n}\right|_{p_{i}}=p_{i}$ if and only if $i \leq 2$. Thus, if $M$ is a maximal subgroup of $G$ such that $\pi(M)=\pi(G)$, then $|M|_{\left\{p_{1}, p_{2}\right\}}=p_{1} p_{2}=|G|_{\left\{p_{1}, p_{2}\right\}}$. Thus if $p_{j}$ is the prime divisor of $|G: M|$, $j \geq 3$. Put $N_{k}=V_{k} \ldots V_{n+1}$ for every $k$ so that $N_{j+1}<M \cap N_{j}<N_{j}$ (the first inequality is strict because $p_{j}$ divides $\left.|M|\right)$. Put $H_{j}=V_{1} \ldots V_{j-1}$ so that $G=H_{j} \ltimes N_{j}$. Set $\pi=\left\{p_{1}, \ldots, p_{j-1}\right\}$ and notice that $\left|H_{j}\right|=|M|_{\pi}$. Let $H$ be a Hall $\pi$-subgroup of $M$ and note that $H$ and $H_{j}$ are conjugate so $G=H \ltimes N_{j}$ and the action of $H$ on $N_{j} / N_{j+1}$ is irreducible. But $M \cap N_{j}$ is $H$-invariant. This is a contradiction. This means that $\pi(M) \neq \pi(G)$ for every $M$ maximal in $G$, and we are done.

Finally, we prove that the $p$-length is bounded.

Proof of Theorem $C$. Notice that the hypothesis is inherited by quotients. Thus, if $G$ is a minimal counterexample, we may assume that $l_{p}(G)=2$ but $l_{p}(G / N)=1$ for every nontrivial normal subgroup $N$ of $G$. By VI.6.9 of [1], for instance, $O_{p^{\prime}}(G)=1, V=O_{p}(G)$ is elementary abelian and is the unique minimal normal subgroup of $G$ and $G=H V$ for some subgroup $H$. Since $l_{p}(G)=2, p$ divides $|H|$ so by hypothesis $H$ is supersolvable. Notice that $O_{p}(H)=1$. Let $L=O_{p^{\prime}}(H), K / L=O_{p}(H / L)$ and let $U$ be a Hall $p^{\prime}$-subgroup of $H$. Notice that $\pi(U V)=\pi(G)$ so by hypothesis $L V \leq U V$ is supersolvable. Write $V=V_{1} \times \cdots \times V_{t}$ with $V_{i}$ cyclic of order $p$ for every $i$ and $L$-invariant. Thus $L$ is isomorphic to a subgroup of the direct product of $t$ copies of the cyclic group of order $p-1$. In particular, all prime divisors of $|L|$ are less than $p$. Now, since $H$ is supersolvable and $K / L$ acts faithfully on $L$, we have a contradiction.

\section{References}

[1] B. Huppert, Endliche Gruppen I, Springer-Verlag, Berlin, Heidelberg, New York, 1967. 
[2] M. Isaacs, "Character Theory of Finite Groups", Dover, New York, 1994.

[3] E. I. Khukhro, V. D. Mazurov, Unsolved Problems in Group Theory: The Kourovka Notebook, No. 19, Sobolev Institute of Mathematics, Novosibirsk, 2018

[4] O. Manz, T. Wolf, "Representations of Solvable Groups", Cambridge University Press, 1993. 